\title{
The use of hyperbaric oxygen to treat actinic rectal fistula after SpaceOAR use and radiotherapy for prostate cancer: a case report
}

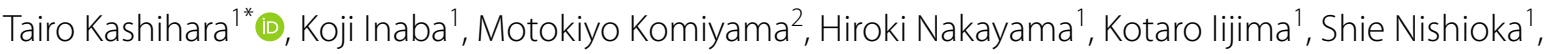 \\ Hiroyuki Okamoto', Nao Kikkawa³, Yuko Kubo³, Satoshi Shima', Satoshi Nakamura', Ayaka Takahashi', \\ Kana Takahashi ${ }^{1}$, Kae Okuma ${ }^{1}$, Naoya Murakami ${ }^{1}$, Hiroshi Igaki ${ }^{1}$, Yuko Nakayama', Arinobu Fukunaga ${ }^{2}$, \\ Yoshiyuki Matsui ${ }^{2}$, Hiroyuki Fujimoto ${ }^{2}$ and Jun Itami ${ }^{1}$
}

\begin{abstract}
Background: In definitive radiation therapy for prostate cancer, the SpaceOAR ${ }^{\circledR}$ System, a hydrogel spacer, is widely used to decrease the irradiated dose and toxicity of rectum. On the other hand, periprostatic abscesses formation and rectal perforation are known as rare adverse effects of SpaceOAR. Nevertheless, there is a lack of reports clarifying the association between aggravation of abscesses and radiation therapy, and hyperbaric oxygen therapy (HBOT) is effective for a peri-SpaceOAR abscess and rectal perforation.

Case presentation: We report a case of a 78-year-old high-risk prostate cancer patient. After SpaceOAR insertion into the correct space, he started to receive external beam radiation therapy (EBRT). He developed a fever, perineal pain and frequent urination after the completion of EBRT, and the magnetic resonance imaging (MRI) revealed a periSpaceOAR abscess. Scheduled brachytherapy was postponed, administration of antibiotics and opioid via intravenous drip was commenced, and transperineal drainage was performed. After the alleviation of the abscess, additional EBRT instead of brachytherapy was performed with MRI-guided radiation therapy (MRgRT). On the last day of the MRgRT, perineal pain reoccurred, and MRI and colonoscopy detected the rectal perforation. He received an intravenous antibiotics drip and HBOT, and fully recovered from the rectal perforation.
\end{abstract}

Conclusions: Our report indicates that EBRT can lead to a severe rectum complication by causing inflammation for patients with a peri-SpaceOAR abscess. Furthermore, HBOT was effective for the peri-SpaceOAR abscess and rectal perforation associated with EBRT.

Keywords: Radiotherapy, Hydrogel spacer, Side effects, Hyperbaric oxygen therapy, MR-guided radiation therapy

*Correspondence: tkashiha@ncc.go.jp

1 Department of Radiation Therapy, National Cancer Center Hospital,

Tsukiji 5-1-1, Chuo-ku, Tokyo, Japan

Full list of author information is available at the end of the article

\section{Background}

A hydrogel spacer is used in radiation therapy (RT) for a variety of cancers to decrease the irradiated dose to the organs at risk (OARs) [1-5]. Furthermore, in pelvic radiation therapy, a hydrogel spacer is used to decrease the rectal dose [6-9]. In definitive RT for prostate cancer, The SpaceOAR ${ }^{\circledR}$ System (Boston Scientific, Marlborough, 
MA, USA), a hydrogel spacer, is widely used to decrease the rectal dose and toxicity $[10,11]$. A prospective randomised study revealed that insertion of SpaceOAR significantly reduced the rectal dose and toxicity and improved bowel/urinary quality of life [12-14]. On the other hand, a patient who developed periprostatic abscess formation after SpaceOAR insertion was reported [15]; however, it was not clear whether SpaceOAR insertion was successful, although the infection improved after percutaneous drainage. Periprostatic abscess is a rare adverse effect of SpaceOAR, and the association between aggravation of abscesses and radiotherapy has not been clarified. Furthermore, the effectiveness of hyperbaric oxygen therapy (HBOT) for peri-SpaceOAR has not been reported. Here, we have presented a case of rectal perforation after peri-SpaceOAR abscess that was successfully treated with HBOT.

\section{Case presentation}

The patient was a 78-year-old prostate cancer patient who had no medical history, except surgical history of goiter and nasal haemangioma. A prostate-specific antigen (PSA) level was $13.89 \mathrm{ng} / \mathrm{mL}$ in a routine evaluation. The clinical stage was T3a. Ultrasound-guided transperineal prostate biopsy revealed Grade Group 4 adenocarcinoma in 1 of 24 specimens. Two months after the biopsy, administration of a luteinizing hormone-releasing hormone agonist was initiated. He opted for external beam RT (EBRT) 46 Gy in 23 fractions combined with high-dose-rate (HDR) brachytherapy 15 Gy in 1 fraction as a definitive treatment. Four months after initiating hormone therapy, SpaceOAR was inserted into the space between the prostate and the rectum, and fiducial markers were inserted into the prostate under local anaesthesia with lidocaine (day 0). The insertion was completed without any side effects, and magnetic resonance imaging (MRI) confirmed that the SpaceOAR was inserted in the correct position (Fig. 1, left). Three weeks after inserting SpaceOAR (day 21), EBRT with computed tomography linear accelerator was initiated. The clinical target volume (CTV) included the prostate, all seminal vesicles, and whole pelvic lymph node regions. The planning target volume (PTV) margin of the whole pelvis was $3 \mathrm{~mm}, 7 \mathrm{~mm}$, and $8 \mathrm{~mm}$ in the RL, SI, and AP directions, respectively. Six days after initiating EBRT (day 27), he developed perineal pain. Owing to increased perineal pain and a diagnosis of urinary tract infection on day 40, antibiotic treatment was initiated. Perineal pain gradually subsided, and he completed oral antibiotic treatment in 1 week (day 47). Four days later (day 51), he experienced perineal pain and frequent urination again; hence, antibiotic treatment was reinitiated. On day 60 , oral administration of opioids was initiated due to increasing perineal pain. The next day (day 61), he developed high fever; thus, MRI was performed for detailed examination. A peri-SpaceOAR abscess was detected on MRI (Fig. 1, middle). Thus, HDR brachytherapy was postponed, administration of antibiotics and opioids via intravenous drip was initiated, and transperineal drainage was performed. Subsequently, the pain gradually subsided and the abscess shrunk slightly on MRI; therefore, intravenous administration of antibiotics changed to oral administration (day 76). Three weeks later (day 97), shrinkage of the abscess and decrease in inflammatory response were confirmed by MRI. Therefore, on day 112, additional RT was initiated. At our conference, EBRT of 20 Gy in 4 fractions was recommended as additional RT instead of HDR monotherapy to decrease the dose
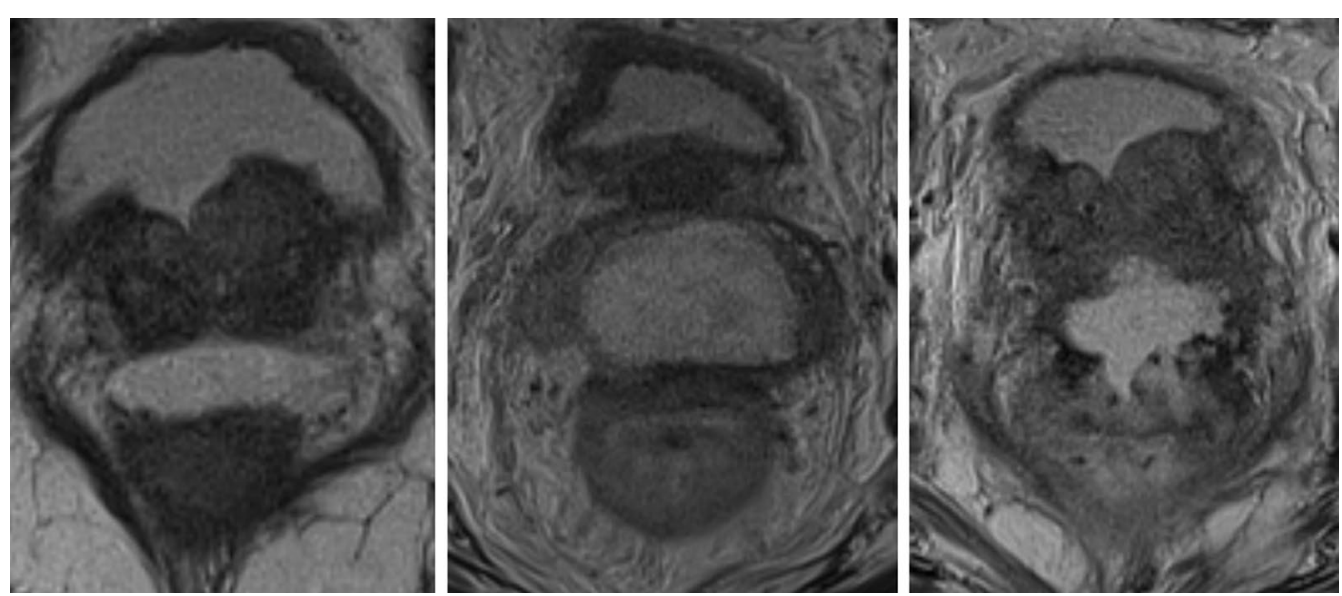

Fig. 1 The changes of MRI findings after the SpaceOAR insertion. MRI taken 1 week after the SpaceOAR insertion (day 7, left). A peri-SpaceOAR abscess (day 61, middle) and a rectal perforation were detected on MRI (day 120, right) 
per fraction. Magnetic resonance-guided RT (MRgRT) with ${ }^{60} \mathrm{Co}$ MRIdian under a magnetic field of $0.345 \mathrm{~T}$ (ViewRay MRIdian System, Oakwood Village, $\mathrm{OH}$ ) was selected to accurately assess intrafractional and interfractional motion of the prostate, seminal vesicles, and OARs (rectum and bladder). The CTV included the prostate and seminal vesicles. The PTV margin was $5 \mathrm{~mm}, 4 \mathrm{~mm}$, and $3 \mathrm{~mm}$ in the RL, SI, and AP directions, respectively. On the last day of MRgRT (day 119), he experienced perineal pain again, and MRI was performed. MRI on day 120 showed aggravated peri-SpaceOAR inflammation and penetration to the rectum was suspected (Fig. 1, right). Colonoscopy was subsequently performed, and penetration of SpaceOAR into the rectum was detected (Fig. 2). To treat rectal perforation, he was kept nothing per os and administration of antibiotics via intravenous drip and intravenous hyperalimentation initiated. He was also transferred to another hospital for HBOT (day 131). HBOT was initiated on day 131. After 24 HBOT sessions for 5 weeks, recovery from rectal perforation was confirmed by colonoscopy, and administration of antibiotics was discontinued. Ten weeks after termination of HBOT, disappearance of a peri-SpaceOAR abscess was confirmed on MRI (day 243).

\section{Discussion and conclusions}

A 78-year-old patient with prostate cancer developed rectal perforation caused by a peri-SpaceOAR abscess and MRgRT (cured by HBOT). To the best of our knowledge, this is the first report showing that HBOT was effective for rectal perforation associated with a periSpaceOAR abscess.

Radiotherapy is one of the most significant treatment modalities in prostate cancer [16-18]. The National
Comprehensive Cancer Network guidelines recommend EBRT + androgen deprivation therapy (ADT) and EBRT + brachytherapy + ADT for the treatment of highrisk prostate cancer [19]. Our patient was scheduled to receive EBRT + HDR brachytherapy + ADT. However, the treatment plan was changed to EBRT + ADT because HDR brachytherapy could cause infection [20] and a large dose per fraction could cause strong inflammation. Furthermore, MRgRT was selected because of its several potential advantages. Murray J et al. [21] reported three advantages of MRgRT-improvement in prostate visibility, monitoring of intrafractional prostate position, and daily adaptive replanning. Owing to these advantages, the margin size of MRgRT in our patient was smaller than that of CT-based RT, as mentioned above.

Radiation-induced intestinal side effects such as bleeding and ulcer are occasionally observed [22-24], but rectal perforation associated with RT is rarely observed. A case of rectal ulceration due to insertion of SpaceOAR into the anterior rectal wall was reported by Teh et al. [25]. However, in our case, SpaceOAR was inserted into the correct space between the prostate and the rectum, confirmed by MRI. A peri-prostate abscess is a rare side effect of SpaceOAR [15]. In our study, after improvement of the peri-SpaceOAR abscess, rectal perforation was detected after EBRT. Rectal perforation could have been caused by not only a peri-SpaceOAR abscess but also inflammation due to EBRT.

HBOT has been reported to be effective for the treatment of abscesses [26-29]. In addition, HBOT is also reported to be effective for the treatment of RT side effects [30-34]. We, therefore, recommended HBOT for the treatment of peri-SpaceOAR abscess and radiationinduced rectal perforation.

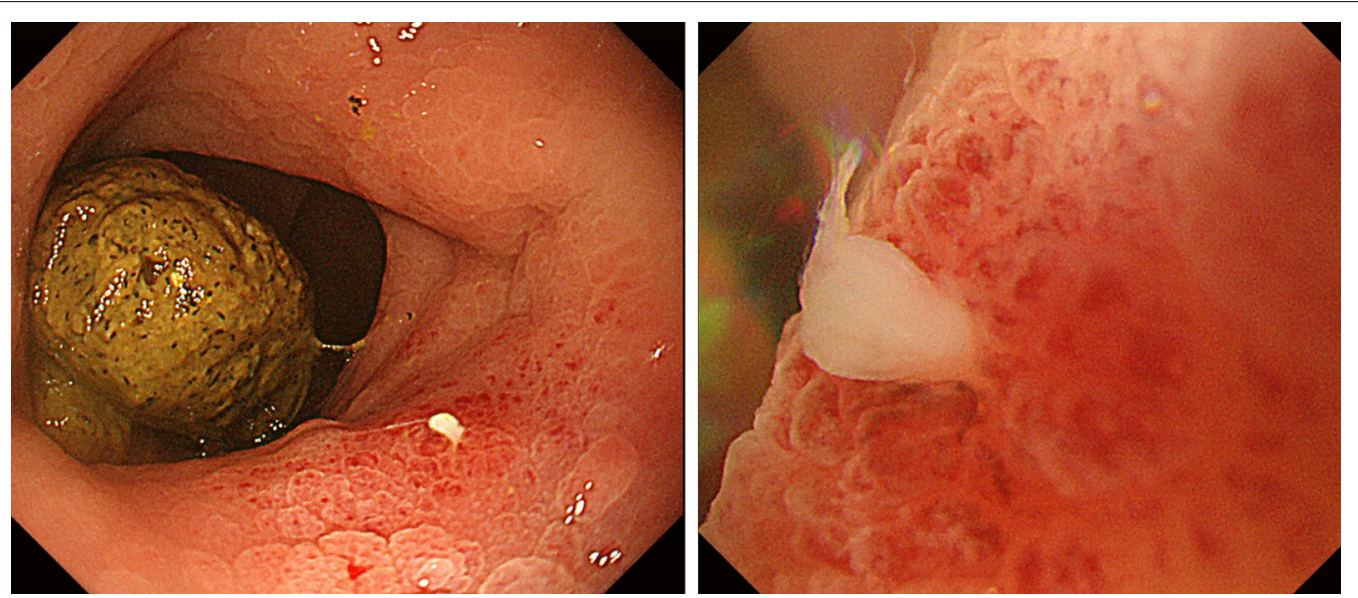

Fig. 2 SpaceOAR penetration into rectum wall detected by colonoscopy. After the penetration of peri-SpaceOAR abscess into rectum wall was suspected on MRI, colonoscopy was performed. SpaceOAR penetrating rectum wall was detected on colonoscopy 
The exacerbation of peri-SpaceOAR abscess and rectal perforation occurred after the resumption of radiotherapy in our patient, but the abscess and rectal perforation resolved after initiating HBOT. The resumption of radiotherapy probably caused decreased blood flow and increased hypoxia, which could reduce endothelial progenitor cell (EPC) homing to the injured rectum area. However, HBOT is known to facilitate EPC trafficking/ homing, thereby promoting wound repair due to angiogenesis [35, 36]. In addition, HBOT elevates hypoxia inducible factor (HIF)-1 and HIF-2 levels in vasculogenic stem/progenitor cells due to increases in reactive oxygen species [36], and this mechanism would also facilitate neovascularization. Furthermore, increasing the oxygen partial pressure can inhibit the growth of anaerobic bacteria and control the infection. HBOT may have helped our patient recover from the abscess and rectal perforation by these mechanisms.

In the management of radiation-induced haemorrhagic cystitis, early initiation of HBOT has been reported to lead to better outcomes [37]. In this previous report, HBOT within 6 months from the onset of haematuria resulted in a better response rate. In our patient, HBOT was initiated within 4 months from the onset of perineal pain, and within 2 weeks from the onset of rectal perforation. It was feared that $\mathrm{HBOT}$ had a cancer-promoting effect and enhanced tumour progression. However, three review articles [38-40] have reported that HBOT does not promote cancer growth. Hence, HBOT was a good treatment option in our case.

Our case report indicates that EBRT can lead to severe rectal complications by causing inflammation in patients with a peri-SpaceOAR abscess. Furthermore, it indicates that HBOT is effective for the treatment of periSpaceOAR abscess and rectal perforation associated with EBRT.

\section{Abbreviations}

ADT: Androgen deprivation therapy; CT: Computed tomographic; CTV: Clinical target volume; EBRT: External beam radiation therapy; HBOT: Hyperbaric oxygen therapy; HDR: High-dose-rate; HIF: Hypoxia inducible factors; LHRH: Leutinizing hormone-releasing hormone; MRgRT: Magnetic resonance imaging-guided radiation therapy; MRI: Magnetic resonance imaging; NCCN: National Comprehensive Cancer Network; OAR: Organ at risk; PTV: Planning target volume; PSA: Prostate-specific antigen; RT: Radiation therapy.
\end{abstract}

\section{Acknowledgements}

We would like to thank Editage (www.editage.jp) for English language editing.

\section{Authors' contributions}

Conception and design of the work: TK, Kl, MK, HN, KI, SN, HO, NK, YK, SS, SN, AT, KT, KO, NM, HI, YN, AF, YM, HF, JI. Supervision and wrote the paper: TK. All authors read and approved the final manuscript.

\section{Funding}

This research received no specific grant from any funding agency in the public, commercial, or not-for-profit sectors.

\section{Availability of data and materials}

The datasets used and/or analysed during the current study are available from the corresponding author on reasonable request.

\section{Ethics approval and consent to participate}

All analyses involving human participants performed in this study were approved by the Institutional Review Board of the National Cancer Center Hospital (approval number, 2017-091) and were in accordance with the ethical standards of the committee and with the 1964 Helsinki declaration and its later amendments or comparable ethical standards.

\section{Consent for publication}

Written informed consent was obtained from the patient for publication of this case report and any accompanying images. A copy of the written consent is available for review by the Editor of this journal.

\section{Competing interests}

Dr. Inaba reports personal fees from Boston Scientific Japan, and grants from Elekta K.K., outside the submitted work. Dr. Nakayama reports personal fees from AstraZeneca, outside the submitted work. Dr. Igaki reports personal fees from Itochu, personal fees from ViewRay Inc., grants from HekaBio, outside the submitted work. Dr. Itami reports grants and non-financial support from KeyJ, personal fees from Alpha Tau, personal fees from ItoChu, outside the submitted work.

\section{Author details}

${ }^{1}$ Department of Radiation Therapy, National Cancer Center Hospital, Tsukiji 5-1-1, Chuo-ku, Tokyo, Japan. ${ }^{2}$ Department of Urological Oncology, National Cancer Center Hospital, Tokyo, Japan. ${ }^{3}$ Department of Radiology, National

Cancer Center Hospital, Tokyo, Japan.

Received: 12 September 2020 Accepted: 3 December 2020

Published online: 14 December 2020

\section{References}

1. Feng Z, Rao AD, Cheng Z, et al. Dose prediction model for duodenum sparing with a biodegradable hydrogel spacer for pancreatic cancer radiation therapy. Int J Radiat Oncol Biol Phys. 2018;102(3):651-9.

2. Rao AD, Feng Z, Shin EJ, et al. A novel absorbable radiopaque hydrogel spacer to separate the head of the pancreas and duodenum in radiation therapy for pancreatic cancer. Int J Radiat Oncol Biol Phys. 2017:99(5):1111-20

3. Kerdsirichairat T, Narang AK, Thompson E, et al. Feasibility of using hydrogel spacers for borderline-resectable and locally advanced pancreatic tumors. Gastroenterology. 2019;157(4):933-5.

4. Struik GM, Pignol JP, Kolkman-Deurloo IK, et al. Subcutaneous spacer injection to reduce skin toxicity in breast brachytherapy: a pilot study on mastectomy specimens. Brachytherapy. 2019;18(2):204-10.

5. Rao AD, Coquia S, De Jong R, et al. Effects of biodegradable hydrogel spacer injection on contralateral submandibular gland sparing in radiotherapy for head and neck cancers. Radiother Oncol. 2018;126(1):96-9.

6. Trifiletti DM, Garda AE, Showalter TN. Implanted spacer approaches for pelvic radiation therapy. Expert Rev Med Devices. 2016;13(7):633-40.

7. Kashihara T, Murakami N, Tselis N, et al. Hyaluronate gel injection for rectum dose reduction in gynecologic high-dose-rate brachytherapy: initial Japanese experience. J Radiat Res. 2019;60(4):501-8.

8. Murakami N, Shima S, Kashihara T, et al. Hyaluronic gel injection into the vesicovaginal septum for high-dose-rate brachytherapy of uterine cervical cancer: an effective approach for bladder dose reduction. J Contemp Brachyther. 2019;11(1):1-7.

9. Murakami N, Nakamura S, Kashihara T, et al. Hyaluronic acid gel injection in rectovaginal septum reduced incidence of rectal bleeding in brachytherapy for gynecological malignancies. Brachytherapy. 2020;19(2):154-61.

10. Hwang ME, Mayeda M, Liz M, et al. Stereotactic body radiotherapy with periprostatic hydrogel spacer for localized prostate cancer: toxicity profile and early oncologic outcomes. Radiat Oncol. 2019;14(1):136. 
11. Wu SY, Boreta L, Wu A, et al. Improved rectal dosimetry with the use of SpaceOAR during high-dose-rate brachytherapy. Brachytherapy. 2018;17(2):259-64.

12. Mariados N, Sylvester J, Shah D, et al. Hydrogel spacer prospective multicenter randomized controlled pivotal trial: dosimetric and clinical effects of perirectal spacer application in men undergoing prostate image guided intensity modulated radiation therapy. Int J Radiat Oncol Biol Phys. 2015;92(5):971-7.

13. Hamstra DA, Mariados N, Sylvester J, et al. Continued benefit to rectal separation for prostate radiation therapy: final results of a phase III trial. Int J Radiat Oncol Biol Phys. 2017;97(5):976-85.

14. Shaikh T, Li T, Handorf EA, et al. Long-term patient-reported outcomes from a phase 3 randomized prospective trial of conventional versus hypofractionated radiation therapy for localized prostate cancer. Int J Radiat Oncol Biol Phys. 2017;97(4):722-31.

15. Hoe V, Yao HH, Huang JG, et al. Abscess formation following hydrogel spacer for prostate cancer radiotherapy: a rare complication. BMJ Case Rep. 2019;12(10):e229143.

16. D'Amico AV, Whittington R, Malkowicz SB, et al. Biochemical outcome after radical prostatectomy, external beam radiation therapy, or interstitial radiation therapy for clinically localized prostate cancer. JAMA 1998;280(11):969-74

17. Parker CC, James ND, Brawley CD, et al. Radiotherapy to the primary tumour for newly diagnosed, metastatic prostate cancer (STAMPEDE): A randomised controlled phase 3 trial. Lancet. 2018;392(10162):2353-66.

18. Kashihara T, Nakamura S, Wakita A, et al. Importance of the site of positive surgical margin in salvage external beam radiation therapy for biochemical recurrence of prostate cancer after radical prostatectomy. Cancer Med. 2018;7(5):1723-30.

19. Mohler JL, Antonarakis ES, Armstrong AJ, et al. Prostate Cancer, Version 2.2019, NCCN clinical practice guidelines in oncology. J Natl Compr Cancer Netw. 2019;17(5):479-505.

20. Emory CL, Montgomery CO, Potter BK, et al. Early complications of high-dose-rate brachytherapy in soft tissue sarcoma: a comparison with traditional external-beam radiotherapy. Clin Orthop Relat Res. 2012;470(3):751-8.

21. Murray J, Tree AC. Prostate cancer-advantages and disadvantages of MR-guided RT. Clin Transl Radiat Oncol. 2019;18:68-73.

22. Fuccio L, Guido A, Andreyev HJ. Management of intestinal complications in patients with pelvic radiation disease. Clin Gastroenterol Hepatol. 2012;10(12):1326-34.

23. Kashihara T, Murakami N, lizumi S, et al. Hemorrhage from ascending colon and gluteal muscle associated with Sorafenib and radiation therapy: radiation dose distribution corresponded with colonoscopy findings and computed tomography images. Pract Radiat Oncol. 2019;9(4):214-9.

24. Weiner JP, Wong AT, Schwartz D, et al. Endoscopic and non-endoscopic approaches for the management of radiation-induced rectal bleeding. World J Gastroenterol. 2016;22(31):6972-86.
25. Teh AY, Ko HT, Barr G, et al. Rectal ulcer associated with SpaceOAR hydrogel insertion during prostate brachytherapy. BMJ Case Rep. 2014:2014:bcr2014206931.

26. Sahin A, Kilic M, Dalgic N. A case report of a 4-year-old boy with intradural spinal cord abscess successfully treated with adjuvant hyperbaric oxygen therapy. Turk Neurosurg. 2019;29(5):789-92.

27. Kutlay M, Colak A, Yildiz S, et al. Stereotactic aspiration and antibiotic treatment combined with hyperbaric oxygen therapy in the management of bacterial brain abscesses. Neurosurgery. 2008:62(Suppl 2):540-6.

28. Cimşit M, Uzun G, Yildiz S. Hyperbaric oxygen therapy as an anti-infective agent. Expert Rev Anti Infect Ther. 2009;7(8):1015-26.

29. Ciodaro F, Gazia F, Galletti B, et al. Hyperbaric oxygen therapy in a case of cervical abscess extending to anterior mediastinum, with isolation of Prevotella corporis. BMJ Case Rep. 2019;12(7):e229873.

30. Hartmann A, Almeling M, Carl UM. Hyperbaric oxygenation (HBO) in the treatment of radiogenic side effects. Strahlenther Onkol. 1996;172(12):641-8

31. Peusch-Dreyer D, Dreyer KH, Müller CD, et al. Management of postoperative radiation injury of the urinary bladder by hyperbaric oxygen ( $\mathrm{HBO})$. Strahlenther Onkol. 1998:174(Suppl 3):99-100.

32. Corman JM, McClure D, Pritchett $R$, et al. Treatment of radiation induced hemorrhagic cystitis with hyperbaric oxygen. J Urol. 2003;169(6):2200-2.

33. Cardinal J, Slade A, McFarland M, et al. Scoping review and meta-analysis of hyperbaric oxygen therapy for radiation-induced hemorrhagic cystitis. Curr Urol Rep. 2018;19(6):38.

34. Ashamalla HL, Thom SR, Goldwein JW. Hyperbaric oxygen therapy for the treatment of radiation-induced sequelae in children. Univ Pa Exp Cancer. 1996;77(11):2407-12

35. Hsu SL, Yin TC, Shao PL, et al. Hyperbaric oxygen facilitates the effect of endothelial progenitor cell therapy on improving outcome of rat critical limb ischemia. Am J Transl Res. 2019;11(4):1948-64.

36. Thom SR. Hyperbaric oxygen - its mechanisms and efficacy. Plast Reconstr Surg. 2011;127(Suppl 1):131S-141S.

37. Chong KT, Hampson NB, Corman JM. Early hyperbaric oxygen therapy improves outcome for radiation-induced hemorrhagic cystitis. Urology. 2005:65(4):649-53.

38. Feldmeier J, Carl U, Hartmann K, et al. Hyperbaric oxygen: does it promote growth or recurrence of malignancy? Undersea Hyperb Med. 2003;30:1-18

39. Daruwalla J, Christophi C. Hyperbaric oxygen therapy for malignancy: a review. World J Surg. 2006;30:2112-31.

40. Moen I, Stuhr LE. Hyperbaric oxygen therapy and cancer-a review. Target Oncol. 2012;7(4):233-42.

\section{Publisher's Note}

Springer Nature remains neutral with regard to jurisdictional claims in published maps and institutional affiliations.
Ready to submit your research? Choose BMC and benefit from:

- fast, convenient online submission

- thorough peer review by experienced researchers in your field

- rapid publication on acceptance

- support for research data, including large and complex data types

- gold Open Access which fosters wider collaboration and increased citations

- maximum visibility for your research: over 100M website views per year

At BMC, research is always in progress.

Learn more biomedcentral.com/submissions 